\title{
Plasma Oscillations and Expansion of an Ultracold Neutral Plasma
}

Scott D. Bergeson

scott.bergeson@byu.edu

S. Kulin

T. C. Killian

S. L. Rolston

Follow this and additional works at: https://scholarsarchive.byu.edu/facpub

Part of the Astrophysics and Astronomy Commons, and the Physics Commons

\section{Original Publication Citation}

S. Kulin, T. C. Killian, S. D. Bergeson and S. L. Rolston, “Plasma oscillations and expansion of an ultracold neutral plasma," Physical Review Letters, 85, 318-321 (2). The original publication of this article can be found at http://link.aps.org/doi/1.113/PhysRevLett.85.318

\section{BYU ScholarsArchive Citation}

Bergeson, Scott D.; Kulin, S.; Killian, T. C.; and Rolston, S. L., "Plasma Oscillations and Expansion of an Ultracold Neutral Plasma" (2000). Faculty Publications. 590.

https://scholarsarchive.byu.edu/facpub/590

This Peer-Reviewed Article is brought to you for free and open access by BYU ScholarsArchive. It has been accepted for inclusion in Faculty Publications by an authorized administrator of BYU ScholarsArchive. For more information, please contact ellen_amatangelo@byu.edu. 


\title{
Plasma Oscillations and Expansion of an Ultracold Neutral Plasma
}

\author{
S. Kulin, T. C. Killian, S. D. Bergeson,* and S. L. Rolston \\ National Institute of Standards and Technology, Gaithersburg, Maryland 20899-8424
}

(Received 24 March 2000)

\begin{abstract}
We report the observation of plasma oscillations in an ultracold neutral plasma. With this collective mode we probe the electron density distribution and study the expansion of the plasma as a function of time. For classical plasma conditions, i.e., weak Coulomb coupling, the expansion is dominated by the pressure of the electron gas and is described by a hydrodynamic model. Discrepancies between the model and observations at low temperature and high density may be due to strong coupling of the electrons.
\end{abstract}

PACS numbers: 52.55.Dy, 32.80.Pj, 52.25.Ub, 52.35.Fp

One of the most interesting features of neutral plasmas is the rich assortment of collective modes that they support. The most common of these is the plasma oscillation [1], in which electrons oscillate around their equilibrium positions and ions are essentially stationary. This mode is a valuable probe of ionized gases because the oscillation frequency depends solely on the electron density.

In an ultracold neutral plasma as reported in [2], the density is nonuniform and changing in time. A diagnostic of the density is thus necessary for a variety of experiments, such as determination of the three-body recombination rate at ultralow temperature [3], and observation of the effects of strong Coulomb coupling [4] in a two-component system. A density probe would also aid in the study of the evolution of a dense gas of cold Rydberg atoms to a plasma [5], which may be an analog of the Mott insulator-conductor phase transition [6].

In this work we excite plasma oscillations in an ultracold neutral plasma by applying a radio frequency (rf) electric field. The oscillations are used to map the plasma density distribution and reveal the particle dynamics and energy flow during the expansion of the ionized gas.

The creation of an ultracold plasma has been described in [2]. Briefly, a few million metastable xenon atoms are laser cooled to approximately $10 \mu \mathrm{K}$. The peak density is about $2 \times 10^{10} \mathrm{~cm}^{-3}$ and the spatial distribution of the cloud is Gaussian with an rms radius $\sigma \approx 220 \mu \mathrm{m}$. These parameters are determined with resonant laser absorption imaging [7]. To produce the plasma, up to $25 \%$ of the atoms are photoionized in a two-photon excitation. Light for this process is provided by a Ti:sapphire laser at $882 \mathrm{~nm}$ and a pulsed dye laser at $514 \mathrm{~nm}(10 \mathrm{~ns}$ pulse length). Because of the small electron-ion mass ratio, the resulting electrons have an initial kinetic energy $\left(E_{e}\right)$ approximately equal to the difference between the photon energy and the ionization potential. In this study we vary $E_{e} / k_{B}$ between 1 and $1000 \mathrm{~K}$. The initial kinetic energy of the ions varies between $10 \mu \mathrm{K}$ and $4 \mathrm{mK}$.

For detection of charged particles, a small de field (about $1 \mathrm{mV} / \mathrm{cm}$ ) directs electrons to a single channel electron multiplier and ions to a multichannel plate detector. The amplitude of the rf field that excites plasma oscillations $F$ varies between $0.2-20 \mathrm{mV} / \mathrm{cm} \mathrm{rms}$. All electric fields are applied to the plasma with grids located above and below the laser-atom interaction region.

In the absence of a magnetic field, the frequency of plasma oscillations is given by $f_{e}=(1 / 2 \pi) \sqrt{e^{2} n_{e} / \epsilon_{0} m_{e}}$ [1]. Here, $e$ is the elementary charge, $n_{e}$ is the electron density, $\boldsymbol{\epsilon}_{0}$ is the permittivity of vacuum, and $m_{e}$ is the electron mass. This relation is most often derived for an infinite homogeneous plasma, but it is also valid in our inhomogeneous system for modes which are localized in regions of near resonant density. Corrections to $f_{e}$ due to finite temperature [8] depend on the wavelength of the collective oscillation, which is difficult to accurately estimate. Such corrections are not expected to be large and will be neglected. We observe plasma oscillations with frequencies from 1 to $250 \mathrm{MHz}$. This corresponds to resonant electron densities $n_{r}$ between $1 \times 10^{4}$ and $8 \times 10^{8} \mathrm{~cm}^{-3}$. The oscillation frequency is sensitive only to $n_{e}$, but, as explained in [2], the core of the plasma is neutral. This implies that plasma oscillations measure electron and ion densities in this region $\left(n_{e}=n_{i} \equiv n\right)$.

Figure 1a shows electron signals from an ultracold neutral plasma created by photoionization at time $t=0$. Some electrons leave the sample and arrive at the detector at about $1 \mu \mathrm{s}$, producing the first peak in the signal. The resulting excess positive charge in the plasma creates a Coulomb potential well that traps the remaining electrons [2]. In the work reported here, typically $90 \%-99 \%$ of the electrons are trapped. Debye shielding maintains local neutrality inside a radius $r_{e}$ beyond which the electron density drops to zero on a length scale equal to $\lambda_{\mathrm{D}}$. The value of $r_{e}$ depends on the fraction of electrons that has escaped, and $\lambda_{\mathrm{D}}$ is the Debye screening length $\lambda_{\mathrm{D}}=\sqrt{\epsilon_{0} k_{B} T_{e} / e^{2} n_{e}}$, where $T_{e}$ is the electron temperature. For our conditions $r_{e} \gtrsim 2 \sigma$ and $\lambda_{\mathrm{D}} \ll \sigma$. As the plasma expands, the depth of the Coulomb well decreases, allowing the remaining electrons to leave the trap. This produces the broad peak at $\approx 25 \mu \mathrm{s}$.

In the presence of an rf field an additional peak appears in the electron signal (Fig. 1a). We understand the generation of this peak as follows: The applied rf field excites plasma oscillations only where the frequency is resonant. 


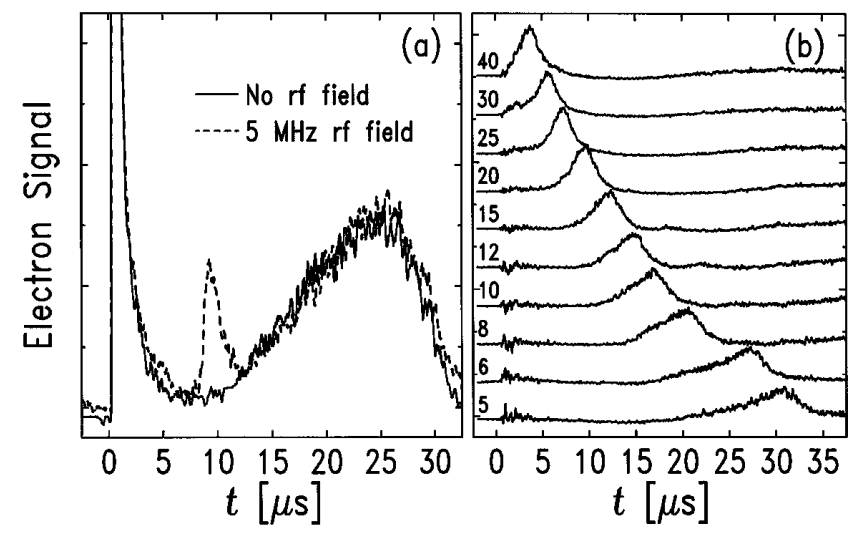

FIG. 1. Electron signals from ultracold plasmas created by photoionization at $t=0$. (a) $3 \times 10^{4}$ atoms are photoionized and $E_{e} / k_{B}=540 \mathrm{~K}$. Signals with and without rf field are shown. The rf field is applied continuously. (b) $8 \times 10^{4}$ atoms are photoionized and $E_{e} / k_{B}=26 \mathrm{~K}$. For each trace, the rf frequency in $\mathrm{MHz}$ is indicated, and the nonresonant response has been subtracted. The signals have been offset for clarity and have been normalized by $F^{2} / n_{r}$. The resonant response arrives later for lower frequency, reflecting expansion of the plasma. For $40 \mathrm{MHz}, n_{r}=2.0 \times 10^{7} \mathrm{~cm}^{-3}$, and for $5 \mathrm{MHz}$, $n_{r}=3.1 \times 10^{5} \mathrm{~cm}^{-3}$.

Energy is thus pumped into the plasma in the shell with the appropriate electron density $\left(n=n_{r}\right)$. The amplitude of the collective electron motion is much less than $\sigma$, but the acquired energy is collisionally redistributed among all the electrons within 10-1000 ns [9], raising the electron temperature. This increases the evaporation rate of electrons out of the Coulomb well, which produces the plasma oscillation response on the electron signal.

The resonant response at a given time $S(t)$ is proportional to the number of electrons in the region where the density equals $n_{r}$. If we make a simple local density approximation and neglect decoherence of the oscillations, $S(t) \propto F^{2} \int d^{3} r n(\mathbf{r}, t) \delta\left[n(\mathbf{r}, t)-n_{r}\right]$. The width in time of the observed signal (Fig. 1a) reflects the density distribution of the sample [10]. At early times when the density is higher than $n_{r}$ almost everywhere, $S(t)$ is negligibly small. As the cloud expands and the density decreases, the response grows because the fraction of the plasma which is in resonance increases. The peak of the response appears approximately when the average density $\bar{n}$ becomes resonant with the rf field. $S(t)$ vanishes when the peak density is less than $n_{r}$.

The resonant response arrives later for lower frequency (Fig. 1b) as expected because $\bar{n}$ decreases in time. Assuming that the plasma density profile remains Gaussian during the expansion, $S(t)$ can be evaluated and its amplitude scales as $F^{2} / n_{r}$. In Fig. $1 \mathrm{~b}$ the data have been normalized by this factor and the resulting amplitudes are similar for all conditions.

By equating $\bar{n}$ to $n_{r}$ when the response peak arrives, we can plot the average plasma density as a function of time (Fig. 2). The data are well described by a self-similar ex-

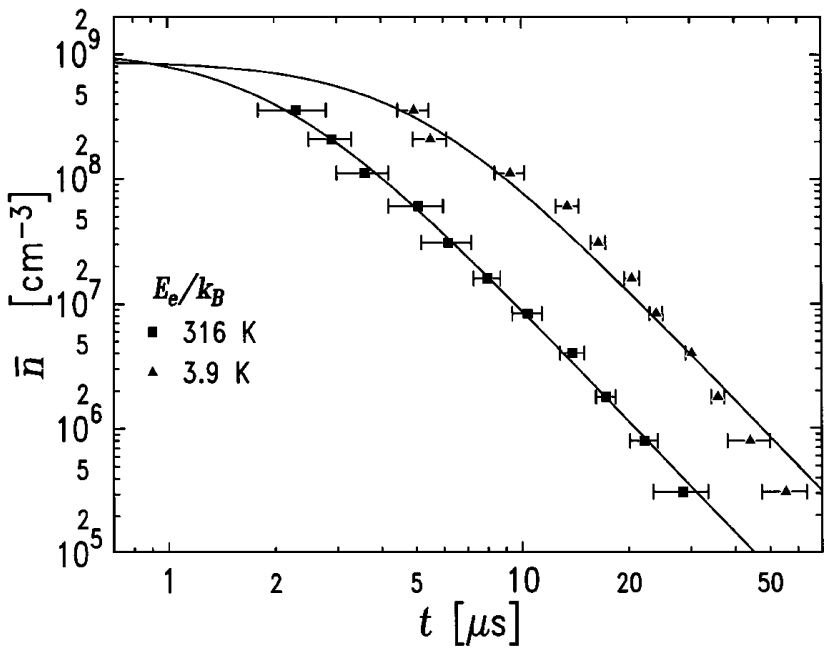

FIG. 2. Expansion of the plasma for $N=5 \times 10^{5}$ photoionized atoms. The expansion is well described by $\bar{n}=$ $N /\left[4 \pi\left(\sigma_{0}^{2}+v_{0}^{2} t^{2}\right)\right]^{3 / 2}$. Horizontal error bars arise from uncertainty in peak arrival times in data such as Fig. 1b. Uncertainty in $N$ is negligible in this data set, but is significant for smaller $N$. The fits are consistently poor at low $E_{e}$, as in the $3.9 \mathrm{~K}$ data.

pansion of a Gaussian cloud, $\bar{n}=N /\left[4 \pi\left(\sigma_{0}^{2}+v_{0}^{2} t^{2}\right)\right]^{3 / 2}$, where $\sigma_{0}$ is the initial rms radius and $v_{0}$ is the rms radial velocity at long times. $N$ is determined independently by counting the number of neutral atoms with and without photoionization. The extracted values of $\sigma_{0}$ are equal to the size of the initial atom cloud. In such an expansion, the average kinetic energy per particle is $3 m v_{0}^{2} / 2$.

Figure 3 shows the dependence of $v_{0}$ on density and initial electron energy. We first discuss data with $E_{e} \geq 70 \mathrm{~K}$, for which the expansion velocities approximately follow $v_{0}=\sqrt{E_{e} / \alpha m_{i}}$, where $m_{i}$ is the ion mass and $\alpha=1.7$ is a fit parameter. For the plasma to expand at this rate, the ions must acquire, on average, a velocity characteristic of the electron energy. This is much greater than the initial ion thermal velocity. Electron-ion equipartition of energy would yield $v_{0}=\sqrt{E_{e} / 3 m_{i}}$, close to the observed value. However, due to the large electron-ion mass difference, this thermalization requires milliseconds [9]. The observed expansion, in contrast, occurs on a time scale of tens of microseconds. One might expect the expansion to be dominated by the Coulomb energy arising from the slight charge imbalance of the plasma, but this energy is about an order of magnitude less than the observed expansion energy. Also, by Gauss' law, it would only be important in the expansion of the non-neutral outer shell of the plasma. The oscillation probe provides information only on the neutral core because it relies on the presence of electrons.

A hydrodynamic model [11], which describes the plasma on length scales larger than $\lambda_{D}$, shows that the expansion is driven by the pressure of the electron gas. The pressure is exerted on the ions by outward-moving electrons that are stopped and accelerated inward in 


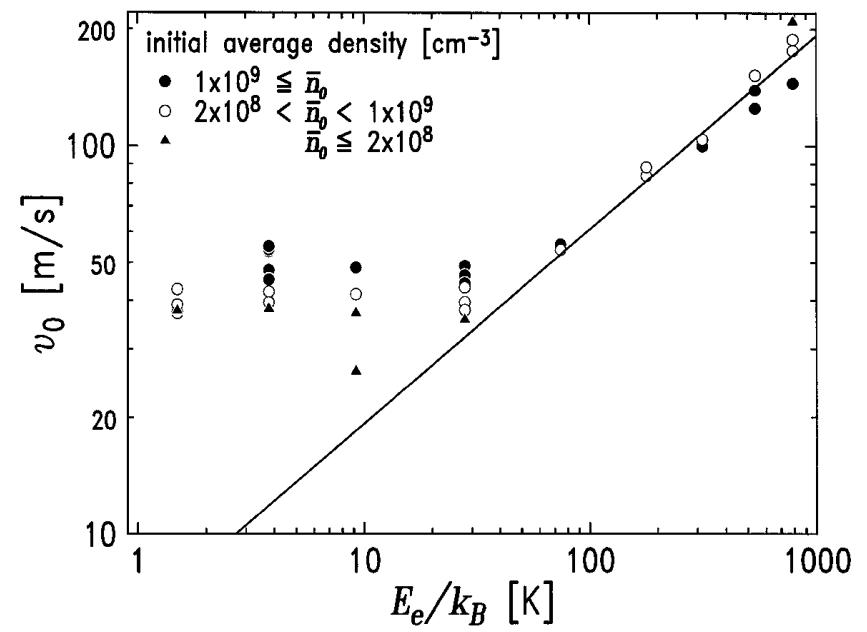

FIG. 3. Expansion velocities $v_{0}$ found from fits to data such as in Fig. 2. The initial average density $\bar{n}_{0}$ varies from $6 \times 10^{6}$ to $2.5 \times 10^{9} \mathrm{~cm}^{-3}$. The solid line, $v_{0}=\sqrt{E_{e} / \alpha m_{i}}$, with $\alpha=$ 1.7 , is a fit to data with $E_{e} / k_{B} \geq 70 \mathrm{~K}$. The behavior of low $E_{e}$ data is discussed in the text. Uncertainty in $v_{0}$ is typically equal to the size of the symbols. There is a $0.5 \mathrm{~K}$ uncertainty in $E_{e} / k_{B}$ reflecting uncertainty in the dye laser wavelength. Note that for $E_{e} / k_{B}<70 \mathrm{~K}, v_{0}$ shows a systematic dependence on $\bar{n}_{0}$.

the trap. For the hydrodynamic calculation, ions and electrons are treated as fluids with local densities $n_{a}(\mathbf{r})$ and average velocities $\mathbf{u}_{a}(\mathbf{r})=\left\langle\mathbf{v}_{a}(\mathbf{r})\right\rangle$. Here, $a$ refers to either electrons or ions, and $\langle\cdots\rangle$ denotes a local ensemble average. Particle and momentum conservation lead to the momentum balance equations

$$
m_{a} n_{a}\left[\frac{\partial \mathbf{u}_{a}}{\partial t}+\left(\mathbf{u}_{a} \cdot \nabla\right) \mathbf{u}_{a}\right]=-\nabla\left(n_{a} k_{B} T_{a}\right)+\mathbf{R}_{a b} .
$$

Here $n_{a} k_{B} T_{a}$ represents a scalar pressure [11]. The ion and electron equations are coupled by $\mathbf{R}_{a b}$, which is the rate of momentum exchange between species $a$ and $b$. The exact form of this term is unimportant for this study, but $\mathbf{R}_{a b}=-\mathbf{R}_{b a}$. Plasma hydrodynamic equations typically have electric and magnetic field terms, but applied and internally generated fields are negligible when describing the expansion.

We can make a few simplifying approximations that are valid before the system has significantly expanded. The directed motion is negligible, so we set $\mathbf{u}_{a} \approx 0$ everywhere. Because $n_{e} \approx n_{i}=n, \partial \mathbf{u}_{e} / \partial t \approx \partial \mathbf{u}_{i} / \partial t$. Because of the small electron mass, the rate of increase of average electron momentum is negligible compared to that of the ions. The electron momentum balance equation then yields $\nabla\left(n k_{B} T_{e}\right) \approx \mathbf{R}_{e i}$, which describes a balance between the pressure of the electron gas and collisional interactions. This is the hydrodynamic depiction of the trapping of electrons by the ions.

In the ion momentum balance equation, we eliminate $\mathbf{R}_{i e}$ using the electron equation, and we drop the pressure term because the ion thermal motion is negligible. Thus $m_{i} n \partial \mathbf{u}_{i} / \partial t \approx-\nabla\left(n k_{B} T_{e}\right)$, which shows that the pressure of the electron gas drives the expansion [12]. This result implies that the ions acquire a velocity of order $\sqrt{k_{B} T_{e} / m_{i}}$, which is in qualitative agreement with the high $E_{e}$ data of Fig. 3. To calculate the expansion velocity more quantitatively, one must consider that as electrons move in the expanding trap, they perform work on the ions and cool adiabatically. The thermodynamics of this process [13] is beyond the scope of this study.

The data in Fig. 3 indicate that about $90 \%$ of the initial kinetic energy of the electrons is transferred to the ions' kinetic energy, $3 m_{i} v_{0}^{2} / 2=3 E_{e} / 2 \alpha$. This does not imply that the temperature of the ions becomes comparable to $E_{e} / k_{B}$ in this process. For the ions, $\mathbf{u}_{i}$ increases, but $m_{i}\left\langle\left|\mathbf{v}_{i}-\mathbf{u}_{i}\right|^{2}\right\rangle$, which measures random thermal motion and thus temperature, is expected to remain small. This follows from slow ion-electron thermalization [9] and correlation between position and velocity during the expansion [14].

We now turn our attention to systems with $E_{e}<70 \mathrm{~K}$ (Fig. 3). They expand faster than expected from an extrapolation of $v_{0}=\sqrt{E_{e} / \alpha m_{i}}$, and thus do not even qualitatively follow the hydrodynamic model. A relative measure of the deviation is $\left(m_{i} v_{0}^{2}-E_{e} / \alpha\right) /\left(E_{e} / \alpha\right)$. Figure 4 shows that the relative deviation increases with increasing electron Coulomb coupling parameter [4], $\Gamma_{e}=\left(e^{2} / 4 \pi \varepsilon_{0} a\right) / k_{B} T_{e}$. Here, $a=(4 \pi n / 3)^{-1 / 3}$ is the Wigner-Seitz radius, $n$ is the peak density at $t=0$, and the temperature is calculated by $3 k_{B} T_{e} / 2=E_{e}$.

The fact that the relative deviation depends only on $\Gamma_{e}$, and that it becomes significant as $\Gamma_{e}$ approaches 1 ,

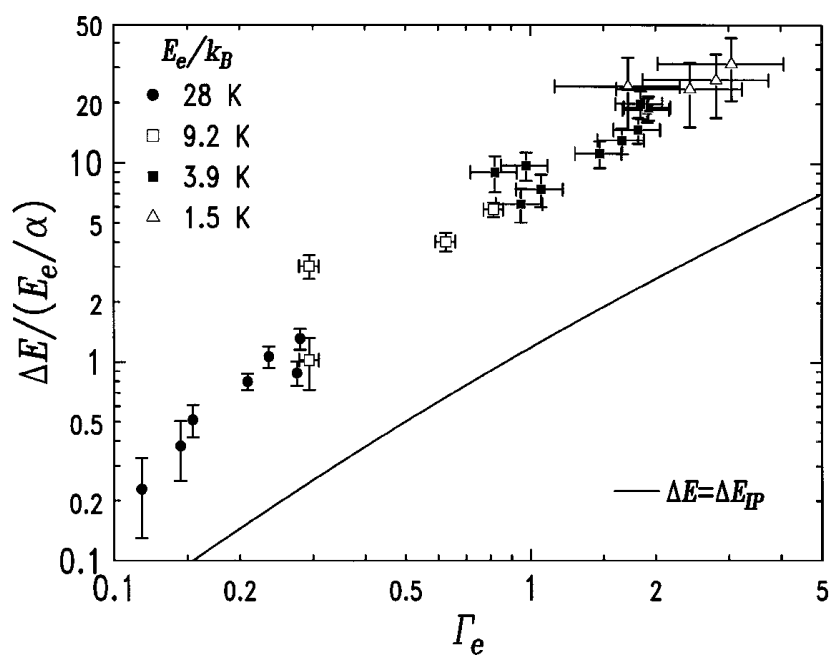

FIG. 4. Excess expansion energy $\Delta E=m_{i} v_{0}^{2}-E_{e} / \alpha$ relative to $E_{e} / \alpha$, as a function of $\Gamma_{e}$, the Coulomb coupling parameter for the electrons at $t=0$. The solid line results from equating $\Delta E$ to the predicted suppression of the atomic ionization potential in the plasma. Horizontal error bars arise from uncertainty in $E_{e}$. Vertical error bars reflect uncertainty in both $E_{e}$ and $v_{0}$. 
suggests that we are observing the effects of strong coupling of the electrons [15]. The hydrodynamic model of the plasma is valid only when $\Gamma_{e} \ll 1$. When $\Gamma_{e} \gtrsim 1$, electron and ion spatial distributions show short range correlated fluctuations that are not accounted for in a smooth fluid description [16]. Correlations between the ion and electron positions, which can be viewed as collisional recombination, would provide the excess kinetic energy observed in the expansion by lowering the potential energy of the plasma. This satisfies overall energy conservation and it may also explain the systematically poor fits of the data for high $\Gamma_{e}$ (see Fig. 2).

Strong coupling is also predicted to alter the relation for the frequency $f_{e}$ [17], with which we extract the plasma density, size, and expansion velocity. The trend of this effect agrees qualitatively with the observed deviation, but knowledge of the wavelength of the collective oscillation is needed for a quantitative comparison.

Other possible explanations for the deviation are related to how the ultracold plasma is created. The $10 \mathrm{~ns}$ duration of the photoionization pulse is long compared to the time required for electrons to move an interparticle spacing. Photoionization late in the pulse thus occurs in the presence of free charges, which will depress the atomic ionization threshold by $\Delta E_{I P} \approx \frac{1}{2} k_{B} T_{e}\left\{\left[\left(3 \Gamma_{e}\right)^{3 / 2}+1\right]^{2 / 3}-1\right\}$ [18]. This effect might increase the electron kinetic energy by $\Delta E_{I P}$ above what has been assumed. However, as shown in Fig. 4, the calculated $\Delta E_{I P}$ is about an order of magnitude smaller than the observed effect. The random potential energy of charged particles when they are created may also yield a greater electron energy than $E_{e}[19]$.

High $\Gamma_{e}$ (high density and low temperature) conditions are desirable for studying the three-body recombination rate in an ultracold plasma. The theory [20] for this process was developed for high temperature, and is expected to break down in the ultracold regime [3]. Measuring or setting an upper limit for the recombination rate is not possible until the dynamics of high $\Gamma_{e}$ systems is understood. We are currently studying this problem with molecular dynamics calculations.

We have shown that plasma oscillations are a valuable probe of the ion and electron density in an ultracold neutral plasma. This tool will facilitate future experimental studies of this novel system, such as the search for other collective modes in the plasma and further investigation of the effects of correlations due to strong coupling.

We thank Lee Collins for helpful discussions and Michael Lim for assistance with data analysis. S. Kulin acknowledges funding from the Alexander-von-Humboldt foundation. This work was funded by the ONR.
* Present address: Department of Physics and Astronomy, Brigham Young University, Provo, UT 84602-4640.

[1] L. Tonks and I. Langmuir, Phys. Rev. 33, 195 (1929).

[2] T. C. Killian, S. Kulin, S. D. Bergeson, L. A. Orozco, C. Orzel, and S. L. Rolston, Phys. Rev. Lett. 83, 4776 (1999).

[3] Y. Hahn, Phys. Lett. A 231, 82 (1997).

[4] S. Ichimaru, Rev. Mod. Phys. 54, 1017 (1982).

[5] S. Kulin, T.C. Killian, S. D. Bergeson, L. A. Orozco, C. Orzel, and S. L. Rolston, in Non-Neutral Plasma Physics III, edited by J. J. Bollinger, R. L. Spencer, and R. C. Davidson (AIP, New York, 1999), p. 367.

[6] Metal-Insulator Transitions Revisited, edited by P.P. Edwards and C.N.R. Rao (Taylor \& Francis, Ltd., London, 1995); G. Vitrant, J. M. Raimond, M. Gross, and S. Haroche, J. Phys. B 15, L49 (1982).

[7] M. Walhout, H. J. L. Megens, A. Witte, and S. L. Rolston, Phys. Rev. A 48, R879 (1993).

[8] D. Bohm and E. P. Gross, Phys. Rev. 75, 1851 (1949).

[9] L. Spitzer, Jr., Physics of Fully Ionized Gases (John Wiley \& Sons, Inc., New York, 1962), Chap. 5.

[10] By applying an rf pulse, we found the response time for excitation and detection of plasma oscillations to be about $1 \mu \mathrm{s}$. This is short compared to the width of $S(t)$, and is probably set by the electron thermalization rate and timeof-flight to the detector.

[11] R.J. Goldston and P.H. Rutherford, Introduction to Plasma Physics (Institute of Physics, Philadelphia, 1995), Chap. 6.

[12] This equation would also describe the ballistic expansion of a single-component cloud of noninteracting particles at temperature $T_{e}$. It also preserves a Gaussian density distribution, which supports our assumption of such a distribution in the data analysis.

[13] G. Manfredi, S. Mola, and M. R. Feix, Phys. Fluids B 5, 388 (1993).

[14] C. Orzel, M. Walhout, U. Sterr, P. S. Julienne, and S. L. Rolston, Phys. Rev. A 59, 1926 (1999).

[15] We expect that the ions are also strongly coupled, although we have no direct evidence for this. The ion-ion thermalization time, equal to that of the electrons, is short, and the low initial ion kinetic energy yields $\Gamma_{i} \gg 1$. Strong coupling of ions is not expected to affect the experiments discussed here.

[16] S. Ichimaru, Statistical Plasma Physics (Addison-Wesley Publishing Co., Reading, MA, 1992), Vol. I, Chap. 7.

[17] G. Kalman, K. I. Golden, and M. Minella, in Strongly Coupled Plasma Physics, edited by H. M. Van Horn and S. Ichimaru (University of Rochester Press, Rochester, 1993), p. 323.

[18] J. C. Stewart and K. D. Pyatt, Jr., Astrophys. J. 144, 1203 (1966); M. Nantel, G. Ma, S. Gu, C. Y. Côté, J. Itatani, and D. Umstadter, Phys. Rev. Lett. 80, 4442 (1998).

[19] E. Eyler and P. Gould (private communication).

[20] P. Mansbach and J. Keck, Phys. Rev. 181, 275 (1969). 